\title{
THE CATHEPSIN-D EXPRESSION IN THE CYTO-INVASIVE STAGES OF COCCIDIA IN INTESTINAL MUCOSA OF CHICKENS, AND THE RELEVANCE OF THIS PROTEINASE FOR INTRACELLULAR PENETRATION AND SPREADING OF COCCIDIA IN HOST TISSUES
}

\author{
Kodousek Rostislava*, Bedrník Petrb ${ }^{\mathrm{a}}$, Černík František ${ }^{\mathrm{b}}$, Kubeš Oldřich ${ }^{\mathrm{c}}$ \\ a*Institute of Pathology, Medical Faculty, Palacký University, 77515 Olomouc, Czech Republic, \\ e-mail: kodousek@tunw.upol.cz
}

b Biofarm, Research Institute for Biopharmacy and Veterinary therapeutics, Pohoří-Chotouň, Jílové by Prague
c Veterinary Station, Olomouc

Recevied July 10, 2001

Key words: Coccidiosis/ Cathepsin-D/ Intracellular penetration/ Spreading factor

The Cathepsin-D proteinase was demonstrated immunohistochemically in cases of intestinal coccidiosis in chickens; its expression concerned almost the cyto-invasive stages of reproductive cycle of several strains of coccidia (merozoites of the II-nd generation and also microgametes). The authors consider Cathepsin-D as a potentional ,penetration/spreading factor", being significant for intracellular invasion and spreading of coccidia into host cells. In diagnostic practice, an immunohistochemical demonstration of Cathepsin-D is a relatively simple and sensitive method, which brings information on the extent of parasitic lesions, and on participation of particular evolutive forms of coccidial life cycle; it may also be useful in proving isolated coccidia in the intestinal epithelium, poorly recognized in routine HE preparation. Some Cathepsin$\mathrm{D}$ protease reactivity in intestinal Cryptosporidiosis is also briefly mentioned. The examined cases of cerebral toxoplasmosis and primary acanthamoebic meningoencephalitis (PAME) showed in above mentioned methods negative results.

\section{INTRODUCTION}

The coccidiosis represent still an actual problem of considerable importance, both in human and in veterinary medicine. From the veterinary point of view, this problem may be of extraordinary importance in cases of massive spreading of coccidiosis in large breedings and poultry farms, leading to far-reaching loss-rates with unfavourable economic consequences.

In the past, we consultatively examined some cases of intestinal coccidiosis (incl. chickens); besides of histopathological fingings and causative agent verification, we also were concerned in mechanisms of spreading of Coccidia in tissues of the host organism. As known, the schizogony with merozoites presents a very effective mode of reproduction, and in some analogy to malaria parasites - makes a pre-condition for further intracellular penetration of invasive forms of parasites with subsequent spreading of infection in host tissues.

The penetration of parasites in host cells, as could be expected, is facilitated by expression of some proteolytic enzymes, and therefore this invasive mechanism has also been followed in this laboratory. We ascertained in histological preparations, that those stages of the coccidian life cycle, which are capable of the intracellular invasivity, show significantly the Cathepsin D - type aspartate proteinase activity. Basing on this finding, Cathepsin-D expression could be considered as some equivalent of the ,penetration or spreading factor" in Coccidia ${ }^{1}$. These invasive Cathepsin D-positive stages of Coccidia have subsequently been identified as merozoites of the II-nd generation and as the microgametes respectively ${ }^{2}$. The both mentioned forms are, as well known, capable of taking part in penetration into the host cells (while macrogametocytes and dif- ferentiated oocysts as well as immature merozoites of the I-st generation were in the mentioned method largely negative). These findings have been furthermore verified on a larger material of experimental intestinal coccidiosis of chickens caused by E. tenella and E. acervulina, and are presented in this communication.

\section{MATERIAL AND METHODS}

Three sets of intestinal coccidiosis in chickens were available for the present studies:

1. Intestinal coccidiosis caused by E. tenella. In this case, an investigation of the appendix samples from chickens was realized.

2. Experimental coccidiosis of chickens induced by $E$. acervulina, E. maxima and E. tenella.

3. Experimental coccidiosis in chickens induced by E. tenella, namely by virulent (1-28 V), and furthermore, by an attenuated strain (1-28 KE).

Sampling was carried out in different time intervals with regard to the course of reproductive cycle. The samples of intestines were fixed in $10 \%$ formalin solution and embedded in paraffin. The sections were stained with haematoxylin and eosin (HE) and with various special methods (PAS-reaction, Müller's colloidal iron reaction, Weigert's method, Giemsa method and Feulgen's nuclear reaction; silver impregnation according to Grocott and Warthin-Starry were also used). Parallel sections were examined by various immunohistochemical methods, viz. for proof of Cathepsin-D, Cathepsin-B, alfa 1-Antichymotrypsin ( $\alpha$ 1-ACT), Cytokeratin, Vimentin, Desmin and E-receptors. Cathepsin D was proved by mo- 
noclonal antibody (DAKO) with peroxidase labeling (dilut. 1:200, incubation time $24 \mathrm{~h}$ or prolonged incubation $48 \mathrm{~h}$ ); the resulting reaction was vizualized by diaminobenzidine (DAB).

\section{RESULTS AND DISCUSSION}

The histopathological findings were typical for intestinal coccidiosis in all above-mentioned groups. However, the extent of lesions of intestinal mucosa was very different, depending upon the strain properties (virulent and attenuated probes) and time factor. Almost regularly, extensive focal lesions in affected mucosa were noticed, massive findings being as result of explosive release and penetration of high quantities of merozoites of the II-nd generation into immediately neighbouring epithelial cells (Fig. 1, 2). This picture appeared as foci of parasite aggregation in certain mucosa segments (basal, lateral and in apical portion of intestinal villi). Various stages of the evolution cycle (merozoites of the I-st and II-nd generation, and at the same time also oocyst precursors) on the oposite sites of neighbouring villi were also noticed. In protracted cases (viz. E. tenella) many coccidial granulomas containing oocysts, localized almost in the lamina propria or in the submucosa, were also present.

By evaluating the histological findings, we used standard criteria for identification of particular stages of reproductive cycle of coccidia as given by Jírovec ${ }^{3}$ and Kaufmann ${ }^{4}$. Basing on immunohistochemical results and comparison of Cathepsin D positive and negative stages of the coccidial reproductive cyclus following conclusion could be made:

(i) immature schizonts - merozoites of the I-st generation - Cathepsin-D negative; (ii) mature schizogony - merozoites of the II-nd generation - Cathepsin-D positive; (iii) microgametocytes - Cathepsin-D positive; (iv) macrogametocytes, oocysts - Cathepsin-D negative.

From these results is evident that only those stages of coccidial reproductive cycle have a proteolytic Cathepsin$\mathrm{D}$ activity, which are capable of the active penetration into host cells - i. e. merozoites of the II-nd generation and microgametocytes. Enzymatic digestion of the cell wall structure enables presumably a parasite to penetrate into cells, where its multiplication (schizogony) occurs; this mode seems to be usual in reproduction of parasitic protozoa and, as mentioned above, is also highly effective.

In connection with an enzymatic penetration factor it would be possible to speculate about the possibility of some preventive blockade of Cathepsin-D activity (by analogy with trypsin blockade carried out in clinical practice) for inhibition of intracellular penetration and spreading of invasive forms of coccidia into the host cells. However, this presumption would demand a relevant experimental verification.

To the contrary of Cathepsin-D positivity in the cytoinvasive coccidial stages, their reactivity for cystein-protei- nase Cathepsin-B showed negative results. However, the Cathepsin-B posivity on merozoites of the I-st generation and the cytoplasm of some affected intestinal mucosal cells was noticed. There was also membrane-bound reactivity on oocysts and their precursors in the case of alfa 1-Antichymotrypsine $(\alpha 1-\mathrm{ACT})$. Some Cathepsin-D reactivity in a case of intestinal cryptosporidiosis was also noticed.

\section{ACKNOWLEDGEMENT:}

The authors express thanks to E. Weigl, (Olomouc) for constructive help (Grant participation, No. MSM 151100002) by preparing this manuscript.

\section{REFERENCES}

1. Kodousek, R., Kubeš, O., Tichý, T. (1998) Coccidiosis in chickens - histopatological findings and the proof of Cathepsin D-positive stages of the reproductive cycle of E. tenella. Presented on the Meeting of North-Moravian patologists, February $4^{\text {th }}$, Olomouc.

2. Bedrník, P. (2000) Personal communication.

3. Jírovec, O. (1948) Parasitologie pro lékaře. Melantrich, Prague.

4. Kaufmann, J. (1996) Parasitic Infection of Domestic Animals. Birkhäuser Verlag

Cathepsin-D in experimental intestinal coccidiosis in chickens.

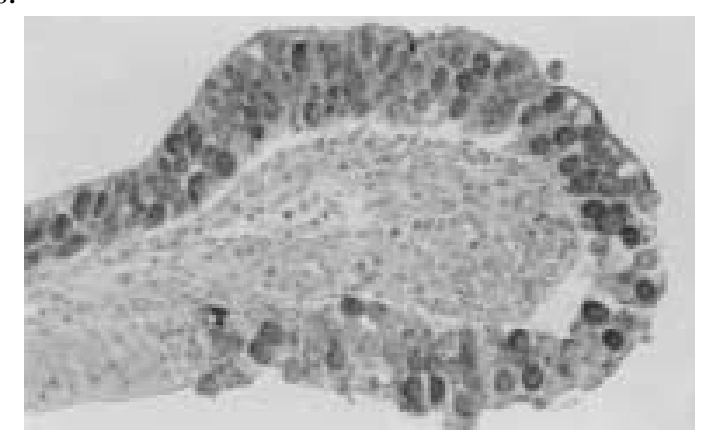

Fig. 1.: Severe involvement of the intestinal mucosa by Cathepsin D-positive intracellular stages of the virulent strain of E. tenella. Orig. magnif. 80x.

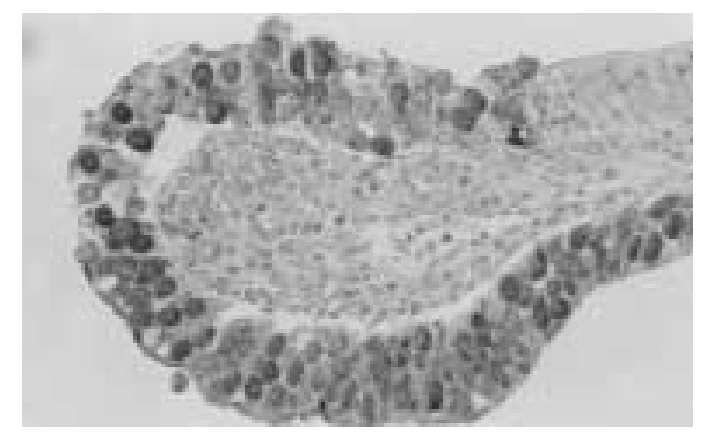

Fig. 2.: Cathepsin D-positive merozoites of the II-nd generation of the strain of E. tenella in intestinal coccidiosis. Orig. magnif. 220x. 